\title{
Towards Ultracold Organic Chemistry: Prospects of Laser Cooling Large Organic Molecules
}

\author{
Maxim V. Ivanov, Felix H. Bangerter ${ }^{\ddagger}$, Paweł Wójcik, Anna I. Krylov* \\ Department of Chemistry, University of Southern California, Los Angeles, California, USA \\ *krylov@usc.edu
}

June 24, 2020

\begin{abstract}
Ultracold organic chemistry enables studies of reaction dynamics and mechanisms in quantum regime. Access to ultracold molecules hinges on the ability to efficiently scatter multiple photons via quasi-closed cycling transitions and, in practice, is complicated by the complex electronic structure of polyatomic molecules. Using equation-of-motion coupledcluster (EOM-CC) calculations, we demonstrate that an alkaline earth metal attached to various aromatic ligands (such as benzene, phenol, cyclopentadienyl and pyrrolide) feature nearly-closed cycling transitions with only a few additional repump lasers. We also show that aromatic ligands such as benzene can accommodate multiple cycling centers in various geometrical configurations and may open new avenues in quantum information science, precision measurements, and ultracold chemistry.
\end{abstract}

\section{Graphical TOC entry:}

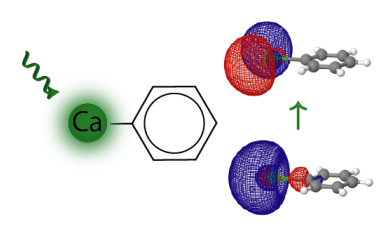


Ultracold molecules provide exciting opportunities to probe chemical reactivity with reagents prepared in a specific quantum state, including vibrational, rotational, spin, and hyperfine levels. ${ }^{1-4}$ The pioneering studies of ultracold chemistry included collisions between atoms, atom/diatomic molecule, and a pair of diatomic molecules. By providing means to study reaction dynamics and mechanism in quantum regime, emerging techniques in molecular physics now open access to more complex chemical systems. Representative examples include a study of the reaction between conformers of 3-aminophenol and laser-cooled $\mathrm{Ca}^{+}, 5$ quantum statecontrolled synthesis of $\mathrm{CaOBa}^{+}, 6$ production of the cold beams of $\mathrm{YbOH}$ via excited-state chemistry, ${ }^{7}$ quantum state-resolved collisions between $\mathrm{OH}$ and $\mathrm{NO}$, between $\mathrm{N}_{2}^{+}$and Rb, between KRb molecules. ${ }^{8-11}$ In addition, access to cold and ultracold molecules is a prerequisite for precision measurements, quantum information storage and simulation. ${ }^{12,13}$

Until only very recently, the laser-cooling was limited to atoms and a handful of diatomic molecules. ${ }^{14-21}$ Recent breakthroughs by Doyle's group, who laser-cooled a series of a polyatomic molecules, from $\mathrm{CaOH},{ }^{22} \mathrm{SrOH}^{23}$ and $\mathrm{YbOH}^{24}$ to much more complex $\mathrm{CaOCH}_{3},{ }^{25}$ inspire an optimism that polyatomic molecules with virtually any degree of complexity can be laser-cooled. Unsaturated hydrocarbons, and aromatic compounds in particular, are at the heart of chemistry. Ability to laser-cool molecules containing aromatic groups would provide a tool to study many important reaction mechanisms in quantum regime.

The challenges in laser-cooling of large polyatomic molecules stem from multiple degrees of freedom and complex electronic structure. In a simple diatomic molecule with atom-like electronic structure, such as CaF, the spontaneous emission returns the excited molecule either to the original ground state or first few excited vibrational levels, thus providing efficient means of laser cooling, with an aid of a few additional repump lasers. In contrast, emission in a large polyatomic molecule often populates numerous vibrational and rotational energy levels, thus requiring an unmanageably large number of repump lasers. That is why viable polyatomic candidates should afford electronic transitions with minimal structural relaxation, i.e., with diagonal Franck-Condon factors (FCFs),${ }^{26-28}$ implying similar equilibrium geometries and normal mode frequencies of the two electronic states involved in the cycling.

One successful strategy of designing a laser-coolable molecule is to attach an alkaline earth metal (such as calcium or strontium) to a ligand that withdraws one of the two valence electrons of the metal creating a strong ionic bond..$^{28-32}$ The second electron remains localized at the 
metal, both in the ground and in the excited states, giving rise to highly diagonal FCFs and thereby closed cycling transitions, if an appropriate number of repump lasers is introduced. The ability to exploit highly localized excitation for optical cycling of such triatomic molecules as $\mathrm{CaOH}, \mathrm{SrOH}$, and $\mathrm{YbOH}$ spur an interest in studying optical cycling schemes in more complex polyatomic molecules. ${ }^{27,28,32-35}$ Using $\mathrm{CaOH}$ as example, the localization of the unpaired electron is facilitated by the locally ionic character of the $\mathrm{Ca}-\mathrm{O}$ bond, and thus various saturated and unsaturated hydrocarbon groups can follow $\mathrm{O}$ atom without significantly deteriorating the FCFs. ${ }^{28}$ A recent demonstration of laser cooling of $\mathrm{CaOCH}_{3}$ proves this concept and suggests that optical cycling and laser cooling of large polyatomic molecules can be as efficient as for much simpler linear species. ${ }^{25}$

Prospects of optical cycling with polyatomic molecules are particularly promising for building novel quantum systems. ${ }^{36}$ It may also pave the way towards (ultra)cold organic chemistry, especially if laser-cooling of aromatic molecules becomes possible. However, considering delocalized nature of the spin/charge distribution in the aromatic systems, ${ }^{37,38}$ it is unclear whether the FCFs remain diagonal when alkaline earth metal is attached to an aromatic ligand.

Following the common design principles, one can construct monovalent alkaline earth metal derivatives of such aromatic molecules as benzene $(\mathrm{Bz})$ and phenol $(\mathrm{Ph})$ as candidates for laser cooling (Figure 1). Alternatively, one can take advantage from (hetero)cyclic $\pi$-conjugated ligands that become aromatic according to Hückel's $4 n+2$ rule, once the electron is transferred from alkaline earth metal to the ligand. Representative examples of the aromatic ligands include cyclopentadienyl $\left(\mathrm{C}_{5} \mathrm{H}_{5}^{-}\right.$or $\left.\mathrm{Cp}\right)$ and pyrrolide $\left(\mathrm{C}_{4} \mathrm{H}_{4} \mathrm{~N}^{-}\right.$or $\mathrm{Py}$ ) anions (Figure 1). By using high-level electronic structure calculations, here we demonstrate that calcium derivatives with various aromatic ligands (Figure 1) may be promising candidates for laser cooling, despite their structural complexity. All electronic structure calculations were performed using the Q-Chem package; ${ }^{39,40}$ the wavefunction analysis was carried out using libwfa library. ${ }^{41}$ The computational details are described in the SI.

We begin with the analysis of the electronic structure of $\mathrm{CaOH}$ - an alkaline earth metal derivative that has been recently laser-cooled. ${ }^{22}$ One of the two valence $s$ electrons of the metal is transferred to the ligand yielding ionically bound $\mathrm{Ca}^{+}$and $\mathrm{OH}^{-}$ions, with Mulliken's charges on $\mathrm{Ca}$ and $\mathrm{O}$ equal to 0.76 and -1.00 , respectively. The interaction between two ions is manifested in the orbital hybridization on the metal atom, which minimizes the repulsion 


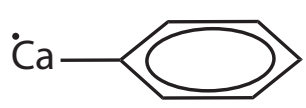

$\mathrm{CaBz}$

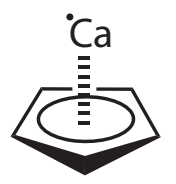

$\mathrm{CaCp}$

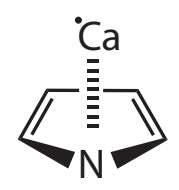

CaPy

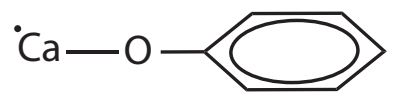

$\mathrm{CaPh}$

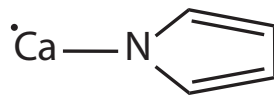

iso-CaPy

Figure 1: Proposed laser-coolable candidates with aromatic ligands

between the unpaired electron and a negatively charged ligand. In the ground $X^{2} \Sigma^{+}$state, the unpaired electron is localized at the metal and occupies the $s \sigma-p \sigma$ hybridized orbital. The transitions from the $X^{2} \Sigma^{+}$state to the $A^{2} \Pi$ and $B^{2} \Sigma^{+}$states can be described as atom-like transitions to $p \pi-d \pi$ and $p \sigma-d \sigma$ hybridized orbitals, respectively (Figure 2A). Accordingly, upon excitation the molecule remains linear, with the most significant structural change being the contraction of the Ca-O bond. Relative to the ground state, Ca-O bond shortens by 0.026 $\AA$ in the $A^{2} \Pi$ state and by $0.024 \AA$ in the $B^{2} \Sigma^{+}$state.

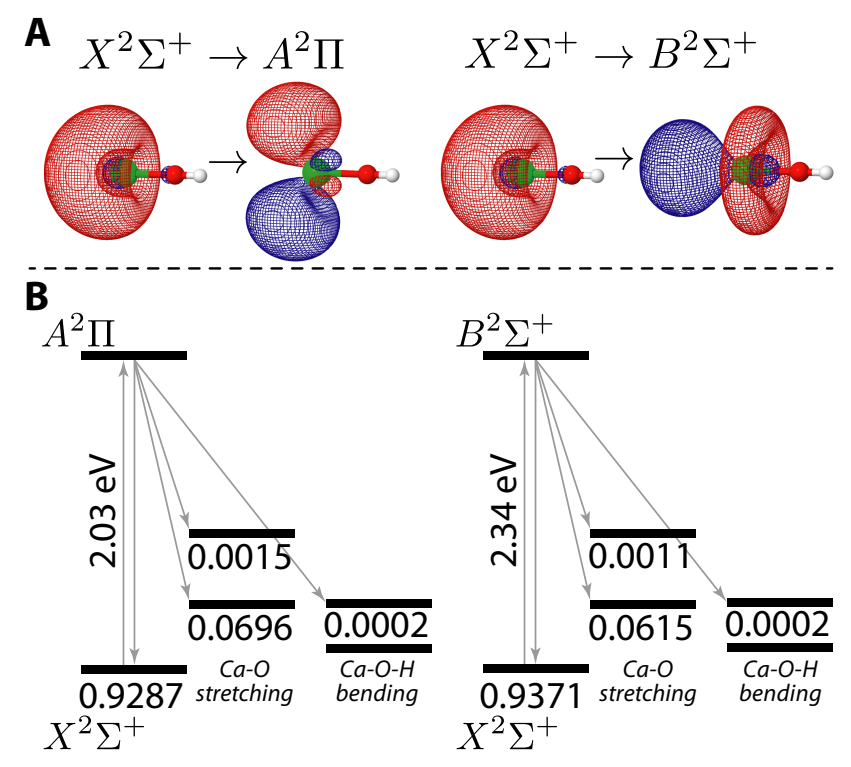

Figure 2: A. Natural transition orbitals for the $X^{2} \Sigma^{+} \rightarrow A^{2} \Pi$ and $X^{2} \Sigma^{+} \rightarrow B^{2} \Sigma^{+}$transitions. B. Optical cycling schemes in $\mathrm{CaOH}$ with indicated FCFs for each vibronic transition. FCFs were computed using the experimental values of the normal mode frequencies.

Our previous benchmarking studies of the optical and structural properties of alkaline earth derivatives showed that equation-of-motion coupled-cluster (EOM-CC) methods ${ }^{42}$ are capable 
of accurately reproducing excitation energies, bond length changes and normal mode frequencies. $^{31}$ Relative errors in the FCFs for the $0 \rightarrow 0$ transition calculated within double-harmonic parallel-mode approximation are in the range of 2-3\%. In $\mathrm{CaOH}$, our calculations yield the FCFs for $A^{2} \Pi(\nu=0) \rightarrow X^{2} \Sigma^{+}(\nu=0)$ and $B^{2} \Sigma^{+}(\nu=0) \rightarrow X^{2} \Sigma^{+}(\nu=0)$ transitions equal 0.9287 and 0.9371 , respectively, in good agreement with the experimentally derived values. Decays into the stretching $\mathrm{Ca}-\mathrm{O}$ and bending $\mathrm{Ca}-\mathrm{O}-\mathrm{H}$ modes are also present and are not negligible (Figure 2B). Although there is no structural relaxation along the bending coordinate, the bending mode is Franck-Condon active due to a noticeable change in the frequency of the bending mode between ground and excited states, causing the decay into the bending mode; this relaxation channel may be further enhanced by anharmonicities.

Our calculations show that the overall picture of the electronic structure is preserved when $\mathrm{Ca}$ is attached directly to the ligand via an in-plane arrangement for all three considered ligands, i.e., $\mathrm{CaBz}, \mathrm{CaPh}$, and $\mathrm{CaPy}$. In particular, the pattern in the electronic excitation spectrum is quite similar to that in $\mathrm{CaOH}$ (Table 1), subject to some qualitative differences. In contrast to linear $\mathrm{CaOH}$, where the first excited state is doubly degenerate $A^{2} \Pi$ state, in $\mathrm{CaBz}, \mathrm{CaPh}$, and $\mathrm{CaPy}$, the $\Pi$ state is split into a $\mathrm{B}_{1} / \mathrm{B}_{2}$ pair separated by $0.02-0.05 \mathrm{eV}$ due to lower symmetry $\left(C_{2 v}\right)$.

Table 1: Excitation energies (in eV) and oscillator strengths (in parenthesis) calculated using EOM-EA-CCSD. Molecules are arranged from more to less symmetric.

\begin{tabular}{l|c|c|c}
\hline \multirow{2}{*}{$\mathrm{CaOH}$} & \multicolumn{2}{|c|}{$A^{2} \Pi$} & $B^{2} \Sigma^{+}$ \\
& \multicolumn{2}{|c|}{$2.03(0.26)$} & $2.34(0.20)$ \\
\hline \multirow{2}{*}{$\mathrm{CaCp}$} & \multicolumn{2}{|c|}{$A^{2} E_{1}$} & $B^{2} A_{1}$ \\
& \multicolumn{2}{|c|}{$1.92(0.19)$} & $2.29(0.16)$ \\
\hline & $A^{2} \mathrm{~B}_{1}$ & $B^{2} \mathrm{~B}_{2}$ & $C^{2} \mathrm{~A}_{1}$ \\
$\mathrm{CaBz}$ & $1.90(0.21)$ & $1.95(0.21)$ & $2.21(0.17)$ \\
$\mathrm{CaPh}$ & $2.07(0.24)$ & $2.09(0.25)$ & $2.34(0.20)$ \\
$\mathrm{CaPy}$ & $2.04(0.23)$ & $2.09(0.23)$ & $2.33(0.19)$ \\
\hline \multirow{2}{*}{ iso-CaPy } & $A^{2} \mathrm{~A}^{\prime \prime}$ & $B^{2} \mathrm{~A}^{\prime}$ & $C^{2} \mathrm{~A}^{\prime}$ \\
& $1.92(0.19)$ & $1.96(0.18)$ & $2.28(0.13)$ \\
\hline
\end{tabular}

Figure 3A shows Dyson orbitals of four lowest electronic states in $\mathrm{CaBz}$ as a representative example of this series. The unpaired electron is mostly localized at the metal and occupies hybridized orbitals that are visually similar to those in $\mathrm{CaOH}$ and other alkaline-earth metal 
derivatives. It is especially surprising, given that the $\mathrm{Ca}-\mathrm{C}$ bond in $\mathrm{CaBz}$ is significantly less ionic than $\mathrm{Ca}-\mathrm{O}$ bond in $\mathrm{CaOH}$-Mulliken's charges on $\mathrm{Ca}$ and $\mathrm{C}$ are 0.47 and -0.42 , respectively. Perhaps, the requirement of a strongly ionic bond is less stringent than previously thought and molecules with a covalent bonding may be laser-coolable as well. This conclusion is consistent with a recent demonstration of laser-cooling of $\mathrm{BaH}$ whose bonding character is much more covalent than in other diatomic molecules (i.e., $\mathrm{CaF}$ and $\mathrm{SrF}$ ). ${ }^{20}$

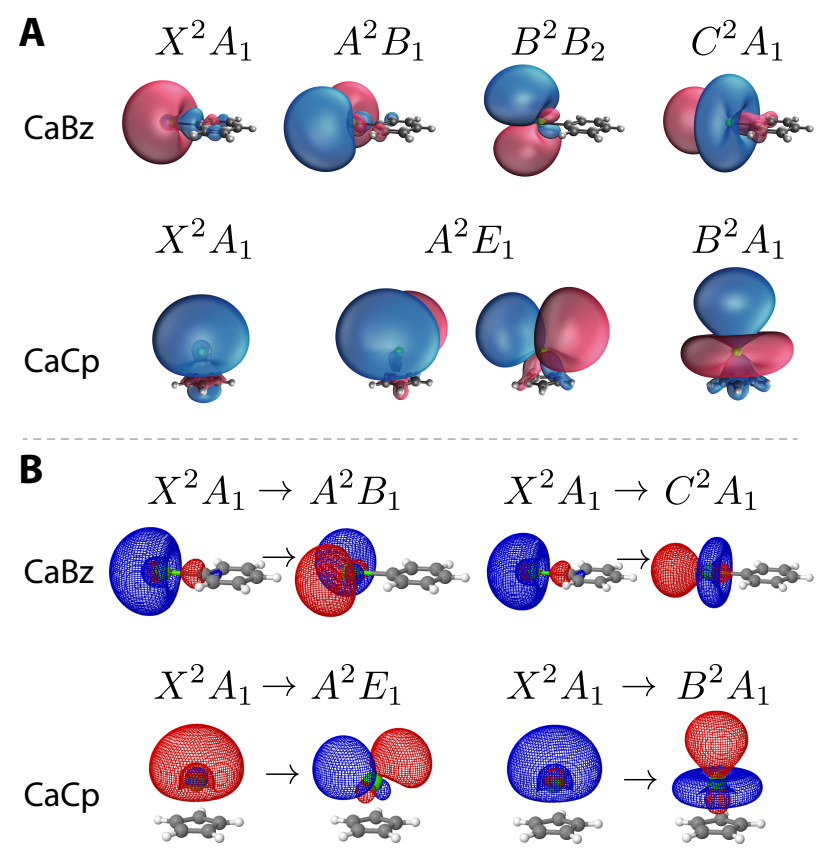

Figure 3: Dyson orbitals (A) and NTOs (B) of CaBz and CaCp plotted with isovalue $=0.03$.

Less ionic character of the $\mathrm{Ca}-\mathrm{C}$ bond manifests itself in a slightly more extended delocalization of the unpaired electron. As Dyson orbitals in Figure 3A show, the unpaired electron distribution spills over the atoms of the ligand to a slightly larger extent than in $\mathrm{CaOH}$. The excitation remains localized at the $\mathrm{Ca}$, with a minor involvement of the directly connected $\mathrm{C}$ atom as shown by NTOs in Figure 3B. Accordingly, the Ca-C bond length changes are slightly larger than those in $\mathrm{CaOH}$ : Ca-C bond is elongated by $0.030 \AA$ in the $A^{2} \mathrm{~B}_{1}$ state and by 0.027 $\AA$ in the $C^{2} \mathrm{~A}_{1}$ state relative to the ground state. The structural relaxation is limited largely to the $\mathrm{Ca}-\mathrm{C}$ bond: the $\mathrm{C}-\mathrm{C}$ bond adjacent to $\mathrm{Ca}-\mathrm{C}$ bond undergoes changes with the magnitudes less than $0.004 \AA$, while the remaining $\mathrm{C}-\mathrm{C}$ bonds are completely unaffected by the excitation.

Estimated FCFs in $\mathrm{CaBz}$ are not as diagonal as in $\mathrm{CaOH}$ and are consistent with the structural relaxation pattern. The FCFs for $A^{2} \mathrm{~B}_{1}(\nu=0) \rightarrow X^{2} \mathrm{~A}_{1}(\nu=0)$ and $C^{2} \mathrm{~A}_{1}(\nu=$ 
$0) \rightarrow X^{2} \mathrm{~A}_{1}(\nu=0)$ are 0.7595 and 0.8849 , respectively. The next largest FCFs are associated with the decays to the first vibrational level of the mode corresponding to the Ca-C stretching (Figure 4A) and are equal to 0.1318 and 0.1055 for the $A^{2} \mathrm{~B}_{1}$ and $C^{2} \mathrm{~A}_{1}$ states, respectively. If both decay channels are addressed by the lasers, a total of 0.8913 and 0.9904 population can be recovered in the $A^{2} \mathrm{~B}_{1} \rightarrow X^{2} \mathrm{~A}_{1}$ and $C^{2} \mathrm{~A}_{1} \rightarrow X^{2} \mathrm{~A}_{1}$ cycling transitions, respectively (Figure $4 \mathrm{~B}$ ). An observation that the FCFs for the $C^{2} \mathrm{~A}_{1} \rightarrow X^{2} \mathrm{~A}_{1}$ transition are more diagonal is consistent with our previous observation of more diagonal FCFs for the corresponding $B^{2} \Sigma^{+} \rightarrow X^{2} \Sigma^{+}$ transition in the linear analogues, ${ }^{31}$ suggesting that this could be general feature of the alkaline earth metal derivatives.

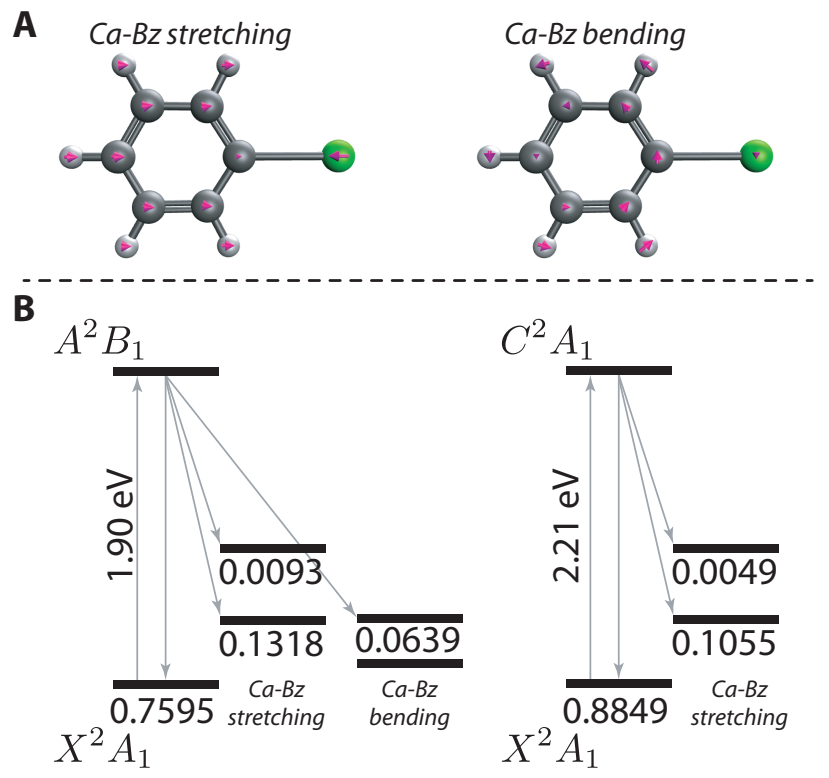

Figure 4: A. Illustration of the normal modes of $\mathrm{CaBz}$ with the largest displacements. B. Optical cycling scheme for the $A^{2} \mathrm{~B}_{1} \rightarrow X^{2} \mathrm{~A}_{1}$ and $C^{2} \mathrm{~A}_{1} \rightarrow X^{2} \mathrm{~A}_{1}$ transitions in $\mathrm{CaBz}$ with indicated FCFs for each vibronic transition.

An additional source of the population decay in the $A^{2} \mathrm{~B}_{1} \rightarrow X^{2} \mathrm{~A}_{1}$ transition is due to the Ca-Bz bending mode, because of the frequency change. With an addition of the third laser, one could recover a total of 0.9552 of the population, which makes this transition viable for optical cycling, despite modestly diagonal FCFs. Structural reorganization in $B^{2} \mathrm{~B}_{2}$ is similar to that in $A^{2} \mathrm{~B}_{1}$ (Figure ?? in $\mathrm{SI}$ ), giving rise to similar FCFs (Table ?? in SI). The other two molecules that we considered, $\mathrm{CaPh}$ and $\mathrm{CaPy}$, show similar patterns in the structural relaxation; their FCFs are given in Table ?? in the SI.

We next turn to the molecules with an out-of-plane arrangement of the cycling center (i.e., 
CaCp and iso-CaPy), which have been previously studied both experimentally and theoretically. ${ }^{43-46}$ Summarizing prior experimental studies, it has been shown that CaCp adopts a $C_{5 v}$ symmetry and features a reach excitation spectrum with extensive vibrational structure due to metal-ring and intra-rings modes, including Jahn-Teller active modes in the doubly degenerate $A^{2} \mathrm{E}_{1}$ state. $^{44}$ Structurally similar iso-CaPy displays a spectrum that is analogous to $\mathrm{CaCp}$, yet exhibits two closely lying but distinct $A$ and $B$ states due to a lower $C_{s}$ symmetry. Despite complex excitation spectrum, the dispersed fluorescence spectra of both CaCp and iso-CaPy are quite simple and feature decays only to the metal-ring stretching mode (Figure 5). The small number of decay channels suggests that the structural relaxation in these molecules is small and, therefore, the FCF matrix is nearly diagonal.
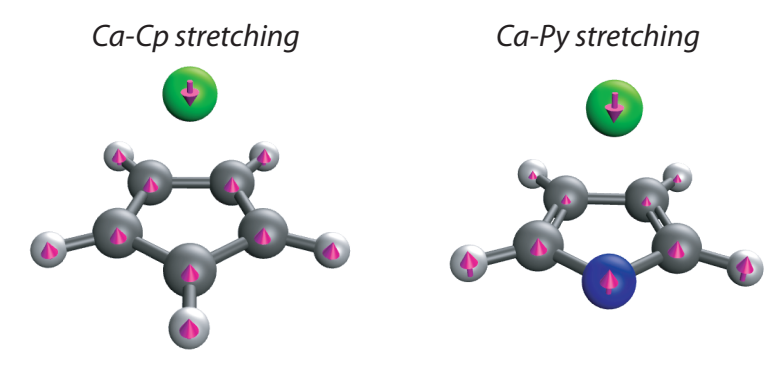

Figure 5: Ca-ring stretching modes of $\mathrm{CaCp}$ and iso-CaPy.

Our calculations reveal that the electronic excitation spectra of $\mathrm{CaCp}$ and iso-CaPy are indeed similar and are consistent with the spectra of other alkaline earth metal derivatives (Table 1). The unpaired electron occupies hybridized atom-like orbitals in each electronic state and electronic transitions are localized at the metal (see example of $\mathrm{CaCp}$ in Figure 3). However, the involvement of the ligand is more significant than in $\mathrm{CaBz}$, as the unpaired electron distribution spills over the aromatic moiety (Figure 3A). An increased bonding between the metal and ring translates into a significant structural relaxation in the excited states: in CaCp the Ca-Cp distance is reduced by $0.070 \AA$ in the $A^{2} \mathrm{E}_{1}$ state and by $0.031 \AA$ in the $B^{2} \mathrm{~A}_{1}$ state, while the intra-ring $\mathrm{C}-\mathrm{C}$ bond changes are less than $0.003 \AA$.

The FCFs are not as diagonal as they are for the in-plane structure, yet with an addition of more repump lasers more than $99 \%$ of the population can be recovered. In the $A^{2} \mathrm{E}_{1} \rightarrow X^{2} \mathrm{~A}_{1}$ cycling transition, 0.5435 of the emission decays back to the ground level, while 0.4480 of the population is distributed across three quanta of the $\mathrm{Ca}-\mathrm{Cp}$ stretching mode (Figure 6). Thus, a total of 0.9915 of the population can be recovered at each cycle with a total of four lasers. 
In the $B^{2} \mathrm{~A}_{1}$ state the magnitudes of the bond length changes are smaller than in $A^{2} \mathrm{E}_{1}$ state. In the $B^{2} \mathrm{~A}_{1} \rightarrow X^{2} \mathrm{~A}_{1}$ cycling transition a total of 0.9914 of the population can be recovered with a total of three lasers (Figure 6). The proposed optical cycling schemes in iso-CaPy are quantitatively similar to those in $\mathrm{CaCp}$ due to similarities in their electronic structure and structural relaxation (see Figure ?? and Table ?? in the SI for details).
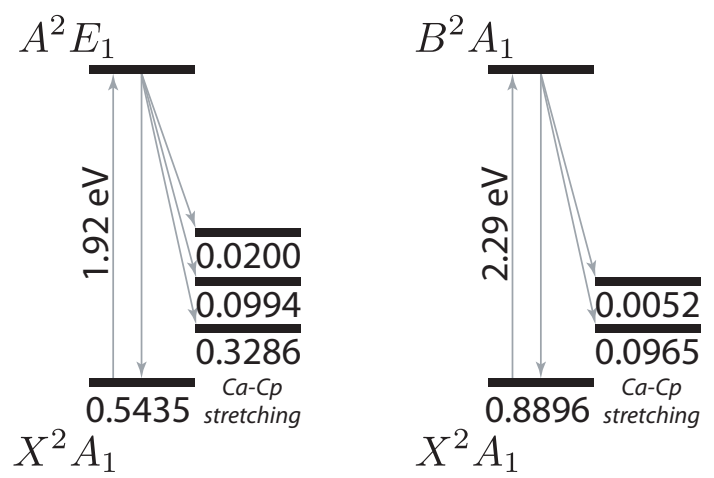

Figure 6: Proposed optical cycling schemes for the $A^{2} \mathrm{E}_{1} \rightarrow X^{2} \mathrm{~A}_{1}$ and $B^{2} \mathrm{~A}_{1} \rightarrow X^{2} \mathrm{~A}_{1}$ transitions in $\mathrm{CaCp}$.

As previously shown, ${ }^{31}$ the electron-withdrawing strength of the ligand has a significant impact on the structural and optical properties of alkaline earth metal derivatives. A judicious choice of the ligand may result in a highly favorable electronic structure and diagonal FCFs. In this context, an alkaline earth metal connected to an aromatic ligand offers a convenient molecular framework suitable for further functionalization. For example, multiple electronwithdrawing ligands of a varied strength can be incorporated into a benzene and cyclopentadienyl moiety of $\mathrm{CaBz}$ and $\mathrm{CaCp}$, respectively (Figure 7). A similar approach has been successfully utilized in the rational design of aromatic superhalogens. ${ }^{47,48}$

A scope of possible applications in QIS, precision measurements and ultracold chemistry can be expanded by incorporating multiple cycling centers into a single molecule. ${ }^{33-35}$ In this context, benzene offers a versatile framework that can host multiple cycling centers with various geometrical configurations. For example, CaBzCa hosts two cycling centers and may exist in three configurations (para, meta, and ortho) with varied separation length between the two centers (Figure 7). Similarly to $\mathrm{CaBz}$, each calcium atom in $\mathrm{CaBzCa}$ hosts unpaired electron giving rise to a diradical character of the electronic wavefunction.

Our EOM-DEA-CCSD calculations show that the electronic structure of para-CaBzCa is quite similar to that of $\mathrm{CaCCCa}$ - a molecule with two calcium atoms separated by the acety- 


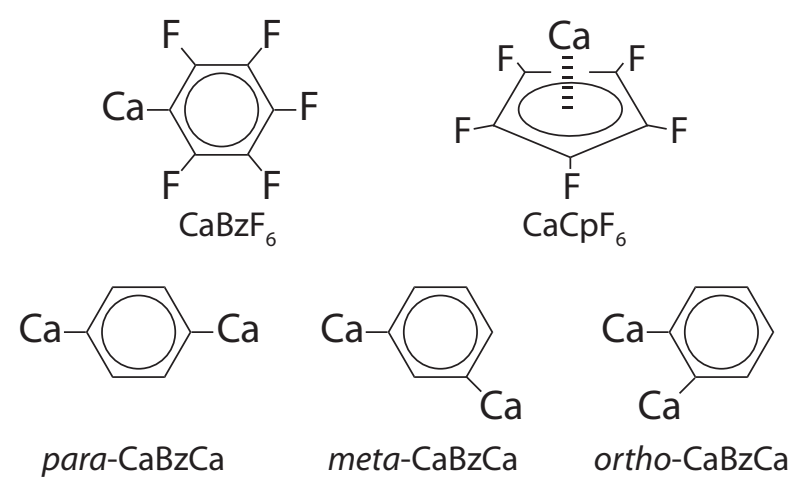

Figure 7: Examples of functionalization in $\mathrm{CaBz}$ and $\mathrm{CaCp}$ (upper panel) and molecules with multiple cycling centers (lower panel).

lene linker. ${ }^{34}$ The ground state of para-CaBzCa features two natural orbitals with near-unity occupations, indicating strong diradical character of the wavefunction (Figure 8A). The antibonding orbital has a slightly higher occupation number, reflecting a considerable role of through-bond interactions, as has been also observed in p-benzyne and CaCCCa. ${ }^{34,49,50}$ In meta- and ortho-CaBzCa the ordering of the two natural orbitals in the ground state switches back to the expected, with bonding orbital below antibonding (Figure ?? in the SI). We therefore conclude that the nature of the interaction between two unpaired electrons is dominated by through-bond interactions in para-CaBzCa and switches to largely through-space in metaand ortho-CaBzCa due to a closer proximity of the two centers.

The structure of the electronic spectrum in para-CaBzCa resembles that of CaCCCa (Figure 8B) ${ }^{34}$ In particular, the lowest electronic states in para-CaBzCa arise from the excitonic splitting between corresponding states in single-centered CaBz. For example, the $\mathrm{B}_{1}$ and $\mathrm{B}_{2}$ states of $\mathrm{CaBz}$ are split by a value of $24-25 \mathrm{meV}$ into the $\mathrm{B}_{1 g} / \mathrm{B}_{2 u}$ and $\mathrm{B}_{2 g} / \mathrm{B}_{1 u}$ pairs (Table ?? in the SI). The value of the excitonic splitting shows a systematic variation with varied $\mathrm{Ca}-\mathrm{Ca}$ distance across three isomers, reflecting the varied change in the through-bond interactions between unpaired electrons (Figure 8B).

In conclusion, here we computationally investigated prospects of laser-cooling large molecules in which an alkaline earth metal is attached to an aromatic ligand. Building upon previous successful frameworks, we designed laser-coolable aromatic molecules by attaching a cycling center to an electron-withdrawing ligand and propose $\mathrm{CaBz}, \mathrm{CaPh}$, and $\mathrm{CaPy}$ as viable candidates. We also exploited (hetero)cyclic $\pi$-conjugated ligands that become aromatic once the 


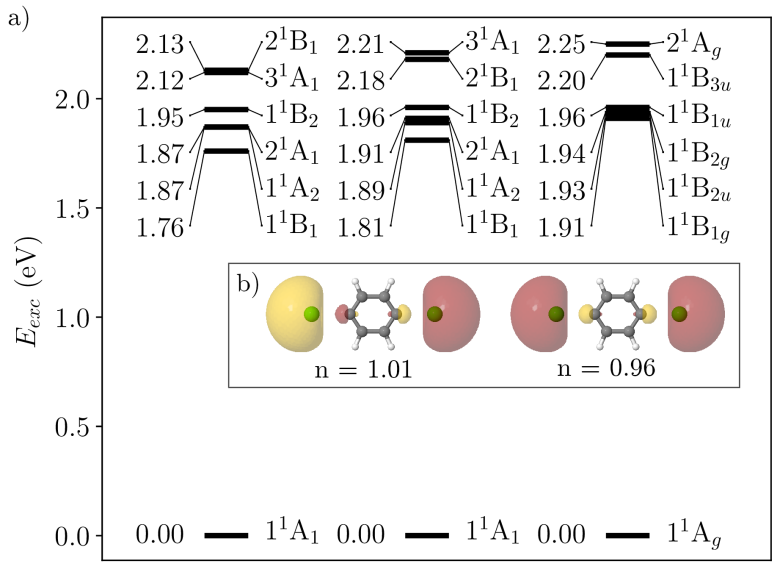

Figure 8: (a) Energy diagrams of ortho-, meta-, and para-CaBzCa calculated using EOMDEA-CCSD/aug-cc-pwCVTZ-PP $[\mathrm{Ca}] /$ cc-pVTZ[C,H]. (b) Natural orbitals (isovalue=0.02) of the ground state in para-CaBzCa.

electron is transferred from the cycling center to the ligand and propose $\mathrm{CaCp}$ and iso-CaPy as candidates. Our primary focus was on the extent of unpaired electron delocalization from the cycling center and how this is translated into the FCFs. We demonstrated that, although the involvement of the ligand in these aromatic molecules is slightly more significant than in triatomic $\mathrm{CaOH}$, the FCFs do not deteriorate significantly. We predict that with an additional few repump lasers, the cycling transition becomes nearly closed, with a total of 96-99\% of the population being recovered. Importantly, the incorporation of aromatic ligands provides means for further functionalization - for example, the optical and structural properties of these molecules can be varied by introducing electron-withdrawing groups. Incorporation of multiple cycling centers further extends the scope of possible applications in QIS, precision measurements, and ultracold chemistry.

\section{Acknowledgments}

This study was funded through the "Molecules Functionalized with Optical Cycling Centers" collaboration, supported by the U.S. Department of Energy (Award DE-SC0019245). We thank Professor Nick Hutzler (Caltech) for helpful discussions.

$\ddagger$ FB current address is Ludwig Maximilian University Munich, Munich, Bavaria, Germany. 


\section{Conflicts of interest}

A.I.K. is the President and a part-owner of Q-Chem, Inc.

Supporting information is available: Computational details; additional results of the EOM-CC calculations (Dyson and natural transition orbitals, equilibrium bond lengths, excitation energies, oscillator strengths). 


\section{References}

[1] Bell, M. T.; Softley, T. P. Ultracold molecules and ultracold chemistry Mol. Phys. 2009, 107, 99-132.

[2] Hutson, J. M. Ultracold chemistry Science 2010, 327, 788-789.

[3] Dulieu, O.; Krems, R.; Weidemüller, M.; Willitsch, S. Physics and chemistry of cold molecules Phys. Chem. Chem. Phys. 2011, 13, 18703-18704.

[4] Quemener, G.; Julienne, P. S. Ultracold molecules under control! Chem. Rev. 2012, 112, 4949-5011.

[5] Chang, Y.-P.; Długołecki, K.; Küpper, J.; Rösch, D.; Wild, D.; Willitsch, S. Specific chemical reactivities of spatially separated 3 -aminophenol conformers with cold $\mathrm{Ca}^{+}$ions Science 2013, 342, 98-101.

[6] Puri, P.; Mills, M.; Schneider, S.; Simbotin, I.; Montgomery, J. A.; Côté, R.; Suits, A. G.; Hudson, E. R. Synthesis of mixed hypermetallic oxide $\mathrm{BaOCa}^{+}$from laser-cooled reagents in an atom-ion hybrid trap Science 2017, 357, 1370-1375.

[7] Jadbabaie, A.; Pilgram, N. H.; Kłos, J.; Kotochigova, S.; Hutzler, N. R. Enhanced molecular yield from a cryogenic buffer gas beam source via excited state chemistry New J. Phys. 2020, 22, 022002.

[8] Hu, M.-G.; Liu, Y.; Grimes, D. D.; Lin, Y.-W.; Gheorghe, A. H.; Vexiau, R.; BouloufaMaafa, N.; Dulieu, O.; Rosenband, T.; Ni, K.-K. Direct observation of bimolecular reactions of ultracold krb molecules Science 2019, 366, 1111-1115.

[9] Kirste, M.; Wang, X.; Schewe, H. C.; Meijer, G.; Liu, K.; van derAvoird, A.; Janssen, L. M. C.; Gubbels, K.; Groenenboom, G. C.; van deMeerakker, S. Y. T. Quantum-state resolved bimolecular collisions of velocity-controlled OH with NO radicals Science $\mathbf{2 0 1 2}$, 338, 1060-1063.

[10] Hall, F. H. J.; Willitsch, S. Millikelvin reactive collisions between sympathetically cooled molecular ions and laser-cooled atoms in an ion-atom hybrid trap Phys. Rev. Lett. 2012, 109, 233202. 
[11] Ospelkaus, S.; Ni, K.-K.; Wang, D.; Miranda, M. H. G. De; Neyenhuis, B.; Quéméner, G.; Julienne, P. S.; Bohn, J. L.; Jin, D. S.; Ye, J. Quantum-state controlled chemical reactions of ultracold potassium-rubidium molecules Science 2010, 327, 853-857.

[12] Kozyryev, I.; Hutzler, N. R. Precision measurement of time-reversal symmetry violation with laser-cooled polyatomic molecules Phys. Rev. Lett. 2017, 119, 133002.

[13] Blackmore, J. A.; Caldwell, L.; Gregory, P. D.; Bridge, E. M.; Sawant, R.; Aldegunde, J.; Mur-Petit, J.; Jaksch, D.; Hutson, J. M.; Sauer, B. E.; Tarbutt, M. R.; Cornish, S. L. Ultracold molecules for quantum simulation: rotational coherences in CaF and RbCs Quantum Sci. Technol. 2019, 4, 014010.

[14] Shuman, E. S.; Barry, J. F.; DeMille, D. Laser cooling of a diatomic molecule Nature 2010, 467,820 .

[15] Barry, J. F.; McCarron, D. J.; Norrgard, E. B.; Steinecker, M. H.; DeMille, D. Magnetooptical trapping of a diatomic molecule Nature 2014, 512, 286.

[16] Hummon, M. T.; Yeo, M.; Stuhl, B. K.; Collopy, A. L.; Xia, Y.; Ye, J. 2D magneto-optical trapping of diatomic molecules Phys. Rev. Lett. 2013, 110, 143001.

[17] Zhelyazkova, V.; Cournol, A.; Wall, T. E.; Matsushima, A.; Hudson, J. J.; Hinds, E. A.; Tarbutt, M. R.; Sauer, B. E. Laser cooling and slowing of CaF molecules Phys. Rev. A 2014, 89, 053416 .

[18] Truppe, S.; Williams, H. J.; Hambach, M.; Caldwell, L.; Fitch, N. J.; Hinds, E. A.; Sauer, B. E.; Tarbutt, M. R. Molecules cooled below the Doppler limit Nat. Phys. 2017, 13, 1173.

[19] Lim, J.; Almond, J. R.; Trigatzis, M. A.; Devlin, J. A.; Fitch, N. J.; Sauer, B. E.; Tarbutt, M. R.; Hinds, E. A. Laser cooled YbF molecules for measuring the electron's electric dipole moment Phys. Rev. Lett. 2018, 120, 123201.

[20] McNally, R. L.; Kozyryev, I.; Vazquez-Carson, S.; Wenz, K.; Wang, T.; Zelevinsky, T. Optical cycling, radiative deflection and laser cooling of barium monohydride (BaH) 2020, page https://arxiv.org/abs/2004.09570; in press; preprint: https://arxiv.org/abs/2004.09570. 
[21] Collopy, A. L.; Ding, S.; Wu, Y.; Finneran, I. A.; Anderegg, L.; Augenbraun, B. L.; Doyle, J. M.; Ye, J. 3D magneto-optical trap of yttrium monoxide Phys. Rev. Lett. 2018, 121, 213201.

[22] Baum, L.; Vilas, N. B.; Hallas, C.; Augenbraun, B. L.; Raval, S.; Mitra, D.; Doyle, J. M. 1D magneto-optical trap of polyatomic molecules Phys. Rev. Lett. 2020, 124, 133201.

[23] Kozyryev, I.; Baum, L.; Matsuda, K.; Augenbraun, B. L.; Anderegg, L.; Sedlack, A. P.; Doyle, J. M. Sisyphus laser cooling of a polyatomic molecule Phys. Rev. Lett. 2017, 118, 173201.

[24] Augenbraun, B. L.; Lasner, Z. D.; Frenett, A.; Sawaoka, H.; Miller, C.; Steimle, T. C.; Doyle, J. M. Laser-cooled polyatomic molecules for improved electron electric dipole moment searches New J. Phys. 2020, 22, 022003; in press; DOI: https://doi.org/10.1088/1367-2630/ab687b.

[25] Mitra, D.; Vilas, N. B.; Hallas, C.; Anderegg, L.; Augenbraun, B. L.; Baum, L.; Miller, C.; Raval, S.; Doyle, J. M. Direct laser cooling of a symmetric top molecule arXiv 2020, page https://arxiv.org/abs/2004.02848; in press; preprint: https://arxiv.org/abs/2004.02848.

[26] Rosa, M. D. Di Laser-cooling molecules Eur. Phys. J. D 2004, 31, 395-402.

[27] Isaev, T. A.; Berger, R. Polyatomic candidates for cooling of molecules with lasers from simple theoretical concepts Phys. Rev. Lett. 2016, 116, 063006.

[28] Kozyryev, I.; Baum, L.; Matsuda, K.; Doyle, J. M. Proposal for laser cooling of complex polyatomic molecules ChemPhysChem 2016, 17, 3641-3648.

[29] Bernath, P. F. Spectroscopy and photochemistry of polyatomic alkaline earth containing molecules Adv. Photochem. 1997, 23, 1-62.

[30] Ellis, A. M. Main group metal-ligand interactions in small molecules: New insights from laser spectroscopy Int. Rev. Phys. Chem. 2001, 20, 551-590.

[31] Ivanov, M. V.; Bangerter, F. H.; Krylov, A. I. Towards a rational design of laser-coolable molecules: Insights from equation-of-motion coupled-cluster calculations Phys. Chem. Chem. Phys. 2019, 21, 19447-19457. 
[32] Augenbraun, B. L.; Doyle, J. M.; Zelevinsky, T.; Kozyryev, I. Molecular asymmetry and optical cycling: Laser cooling asymmetric top molecules 2020, page https://arxiv.org/abs/2001.11020; in press; preprint: https://arxiv.org/abs/2001.11020.

[33] O’Rourke, M. J.; Hutzler, N. R. Hypermetallic polar molecules for precision measurements Phys. Rev. A 2019, 100, 022502.

[34] Ivanov, M. V.; Gulania, S.; Krylov, A. I. Two cycling centers in one molecule: Communication by through-bond interactions and entanglement of the unpaired electrons J. Phys. Chem. Lett. 2020, 11, 1297-1304; in press.

[35] Kłos, J.; Kotochigova, S. Prospects for laser cooling of polyatomic molecules with increasing complexity Phys. Rev. Res. 2020, 2, 013384.

[36] Zhao, C.; Yu, S.; Shin, A.; Long, X.; Atallah, T.; Caram, J.; Campbell, W. Molecules functionalized with optical cycling centers Bull. Am. Phys. Soc. 2019, 64.

[37] Heckmann, A.; Lambert, C. Organic mixed-valence compounds: a playground for electrons and holes Angew. Chem., Int. Ed. 2012, 51, 326-392.

[38] Wang, D.; Talipov, M. R.; Ivanov, M. V.; Rathore, R. Energy gap between the poly-pphenylene bridge and donor groups controls the hole delocalization in donor-bridge-donor wires J. Am. Chem. Soc. 2016, 138, 16337-16344.

[39] Shao, Y.; Gan, Z.; Epifanovsky, E.; Gilbert, A.T.B.; Wormit, M.; Kussmann, J.; Lange, A.W.; Behn, A.; Deng, J.; Feng, X., et al. Advances in molecular quantum chemistry contained in the Q-Chem 4 program package Mol. Phys. 2015, 113, 184-215.

[40] Krylov, A. I.; Gill, P. M. W. Q-Chem: An engine for innovation WIREs: Comput. Mol. Sci. 2013, 3, 317-326.

[41] Plasser, F.; Wormit, M.; Dreuw, A. New tools for the systematic analysis and visualization of electronic excitations. I. Formalism J. Chem. Phys. 2014, 141, 024106-13.

[42] Krylov, A. I. Equation-of-motion coupled-cluster methods for open-shell and electronically excited species: The hitchhiker's guide to Fock space Annu. Rev. Phys. Chem. 2008, 59, $433-462$. 
[43] Ortiz, J. V. Electron propagator theory of the ground and excited states of $\mathrm{CaC}_{5} \mathrm{H}_{5} \mathrm{~J}$. Am. Chem. Soc. 1991, 113, 3593-3595.

[44] Robles, E. S. J.; Ellis, A. M.; Miller, T. A. Electronic spectroscopy of jet-cooled halfsandwich organometallic complexes $\mathrm{CaC}_{5} \mathrm{H}_{5}, \mathrm{CaC}_{5} \mathrm{H}_{4} \mathrm{CH}_{3}$, and $\mathrm{CaC}_{4} \mathrm{H}_{4} \mathrm{~N}$ J. Am. Chem. Soc. 1992, 114, 7171-7183.

[45] Cerny, T. M.; Williamson, J. M.; Miller, T. A. Rotationally resolved electronic spectra of the "half-sandwich" organometallic radical, $\mathrm{CaC}_{5} \mathrm{H}_{5}$ J. Chem. Phys. 1995, 102, 2372-2378.

[46] Chan, W. T.; Hamilton, I. P. Geometries and vibrational frequencies for calcium and strontium radical salts of $\mathrm{C}_{5} \mathrm{H}_{5}, \mathrm{C}_{5} \mathrm{H}_{4} \mathrm{CH}_{3}, \mathrm{C}_{4} \mathrm{H}_{4} \mathrm{~N}$, and $\mathrm{BH}_{4}$ Chem. Phys. Lett. 2000, 316, $171-175$.

[47] Giri, S.; Child, B. Z.; Jena, P. Organic superhalogens Comp. Phys. Comm. 2014, 15, 2903-2908.

[48] Zhou, F. Q.; Zhao, R.-F.; Li, J.-F.; Xu, W.-H.; Li, C.-C.; Luo, L.; Li, J.-L.; Yin, B. Constructing organic superacids from superhalogens is a rational route as verified by DFT calculations Phys. Chem. Chem. Phys. 2019, 21, 2804-2815.

[49] Baldridge, K. K.; Buttersby, T. R.; Clark, R. V.; Siegel, J. S. Does $\pi-\sigma-\pi$ through bond coupling significantly increase C-C bond lengths? J. Am. Chem. Soc. 1997, 119, $7048-7054$.

[50] Orms, N.; Rehn, D. R.; Dreuw, A.; Krylov, A. I. Characterizing bonding patterns in diradicals and triradicals by density-based wave function analysis: A uniform approach $J$. Chem. Theory Comput. 2018, 14, 638-648. 